\title{
Targeted therapies in the management of renal cell carcinoma: role of bevacizumab
}

\author{
Bernard Escudier' \\ Jan Cosaert ${ }^{2}$ \\ Sangeeta Jethwa ${ }^{2}$ \\ 'Unité Immunothérapie, Institut \\ Gustave Roussy, Villejuif, France; \\ ${ }^{2}$ F. Hoffmann-La Roche Ltd, Basel, \\ Switzerland
}

\begin{abstract}
Bevacizumab (10 mg/kg every 2 weeks), in combination with interferon alpha-2a (IFN), is an effective option for first-line therapy for advanced and/or metastatic renal cell carcinoma (RCC). Two phase III trials clearly show significant improvements in progression-free survival and response rate in patients with treatment-naïve metastatic RCC receiving bevacizumab combined with IFN compared with IFN. The dose of IFN, which was initiated at 9 MIU 3 times a week in these trials, can be reduced to effectively manage IFN-related side effects without compromising the efficacy of bevacizumab plus IFN. Bevacizumab has good tolerability with manageable side effects, both alone and in combination with other agents; the tolerability profile of bevacizumab in combination with IFN is consistent with the well-characterized and well-established profiles of these therapies. The tolerability of bevacizumab combined with IFN and the flexibility to manage IFN-related side effects are important considerations when selecting first-line therapy. With a number of options now available for RCC therapy, optimizing their use is a key consideration in improving patient benefit.
\end{abstract}

Keywords: bevacizumab (Avastin ${ }^{\circledR}$ ), interferon alpha, efficacy, tolerability, renal cell carcinoma (RCC), low-dose interferon

\section{Bevacizumab development}

Vascular endothelial growth factor (VEGF) is a ligand that primarily acts upon vascular endothelial cells and is produced by a number of different cell types (Leung et al 1989; Ferrara et al 2003; Ferrara 2004). Binding of VEGF to its main receptor, VEGF receptor-2, results in an array of biological effects including increased vascular permeability, induction of proliferation and migration of endothelial cells (angiogenesis), and promotion of the survival of immature endothelial cells via apoptosis inhibition. As such it is a key pro-angiogenic molecule, playing a critical role in the angiogenesis essential to physiological processes such as embryogenesis and skeletal growth (Carmeliet et al 1996; Ferrara et al 1998; Gerber et al 1999; Bloch et al 2000). However, in adulthood, the physiological role of VEGF is limited and includes processes such as wound healing and the female reproductive function.

VEGF is a key mediator of angiogenesis in cancer (Carmeliet 2005). When oxygen and nutrient levels are insufficient to continue proliferation, tumors become hypoxic and further growth is prevented. To facilitate further tumor growth, angiogenesis is essential and the production of growth factors such as VEGF by the tumor in response to cellular hypoxia is a key mediator. VEGF is continuously expressed throughout the development of many tumor types, and is the only angiogenic factor known to be present throughout the entire tumor life cycle (Folkman 2005). Despite the resulting tumor-associated vasculature being abnormal and inefficient, it is vital for tumor survival and proliferation.

Targeting tumor vasculature as a means of therapeutic intervention was first proposed by Folkman in 1971 (Folkman 1971; Folkman et al 1971). The identification 
and characterization of VEGF in the 1980s (Senger et al 1983; Senger et al 1986; Ferrara and Henzel 1989) made this mode of therapy a real prospect, and targeting VEGF as a means of preventing angiogenesis led to the development of bevacizumab (Avastin ${ }^{\circledR}$ ). Bevacizumab is a recombinant humanized monoclonal immunoglobulin G1 (IgG1) monoclonal antibody $(\mathrm{mAb})$ that directly inhibits VEGF. It was developed from the murine anti-VEGF mAb after A4.6.1, which was shown to be effective in suppressing the growth of tumour xenografts in animal models (Kim et al 1993; Warren et al 1995). However, as a foreign protein, A4.6.1 would be unsuitable for use in humans because of the immune response it would invoke. In addition to potentially causing allergic reactions, its bioavailability and half-life would be greatly limited. Therefore, the murine anti-VEGF mAb was humanized by site-directed mutagenesis to produce bevacizumab, which is $93 \%$ human and $7 \%$ murine and recognizes all major isoforms of human VEGF with a similar binding affinity to the murine antibody $\left(\mathrm{Kd}=8 \times 10^{-10} \mathrm{M}\right)$ (Presta et al 1997). Bevacizumab binding to VEGF sterically hinders VEGF ligation to its receptors on vascular endothelial cells (Kim et al 1992).

A number of effects on tumor vasculature are observed with sustained inhibition of VEGF using bevacizumab. Regression of existing tumor microvasculature, normalization of surviving tumor vasculature and inhibition of the formation of new vasculature are all seen with bevacizumab therapy (Presta et al 1997; Willett et al 2004). It has also been suggested that bevacizumab may reverse tumor-associated immune suppression as well as improving concomitant drug delivery into the tumor (Jain 2002; Wildiers et al 2003; Tong et al 2004). The requirement for continued VEGF inhibition for continued tumor growth inhibition is highlighted by studies showing that withdrawal of anti-VEGF therapy results in rapid regrowth of tumor vasculature, suggesting that anti-VEGF therapy should be continued until disease progression (Mancuso et al 2006).

Initial clinical trials of bevacizumab showed that it was well tolerated when administered alone and in combination with various chemotherapy regimens to patients with solid tumors (Gordon et al 2001; Margolin et al 2001). Subsequent phase II trials established promising efficacy and tolerability when used in combination with standard chemotherapy regimens for colorectal cancer (CRC) and non-small cell lung cancer (NSCLC) (Kabbinavar et al 2003; Johnson et al 2004). Based on data from a phase III trial of irinotecan/5-fluorouracil/leucovorin (IFL) plus placebo vs IFL plus bevacizumab in patients with metastatic CRC, which demonstrated a significant overall survival (OS) benefit for the combination, the US Food and Drug Administration (FDA) approved bevacizumab as part of first-line treatment for metastatic CRC in February 2004, making bevacizumab the first anti-VEGF therapy to be approved for clinical use (Ferrara et al 2005). Bevacizumab has now also been approved as part of first-line therapy for metastatic breast cancer and advanced NSCLC in many countries worldwide. As a result of extensive clinical experience from more than 10 completed phase III trials and more than 250,000 patients treated worldwide, bevacizumab's tolerability profile is well characterized. In December 2007, the European Medicines Agency (EMEA) approved bevacizumab, in combination with interferon alpha-2a (IFN), as first-line treatment for patients with advanced and/or metastatic renal cell carcinoma (RCC) (Roche Media News 2007).

\section{RCC: disease overview}

RCC represents 3\% of all cancers (Ferlay et al 2007; Jemal et al 2008) and approximately $85 \%$ of all renal tumors (Motzer et al 1996), more than 200,000 cases of RCC are diagnosed annually worldwide. RCC affects more men than women (approximately in a ratio of $2: 1$ ) and its incidence peaks in people aged 60-80 years (Corgna et al 2007). Improved imaging techniques and more incidental findings may partly be responsible for an observed increase in the overall incidence of RCC in recent years (Lipworth et al 2006; Corgna et al 2007). Over 100,000 deaths are attributed to RCC each year (Ferlay et al 2007; Jemal et al 2008) although mortality rates appear to be stabilizing, and in some cases declining, following steady increases in mortality rate over the past few decades (Bosetti et al 2008; Karim-Kos et al 2008; Levi et al 2008). The reasons for this improvement are currently undetermined.

The proximal renal tubular epithelium is the primary site of origin for RCC, which can be categorized into 4 main subtypes (Cheville et al 2003): clear cell, papillary, chromophobic and collecting duct. Clear cell RCC accounts for approximately $85 \%$ of all RCC tumors (Figure 1) (Cheville et al 2003).

Major risk factors linked with the development of RCC include smoking, obesity, hypertension and chronic dialysis (Lipworth et al 2006), but RCC occurs in both sporadic and hereditary forms, with at least 4 hereditary syndromes recognized (Linehan et al 2003; Vira et al 2007): von Hippel-Lindau (VHL) syndrome (characterized by mutations/deletions of the $V H L$ tumor suppressor gene); 


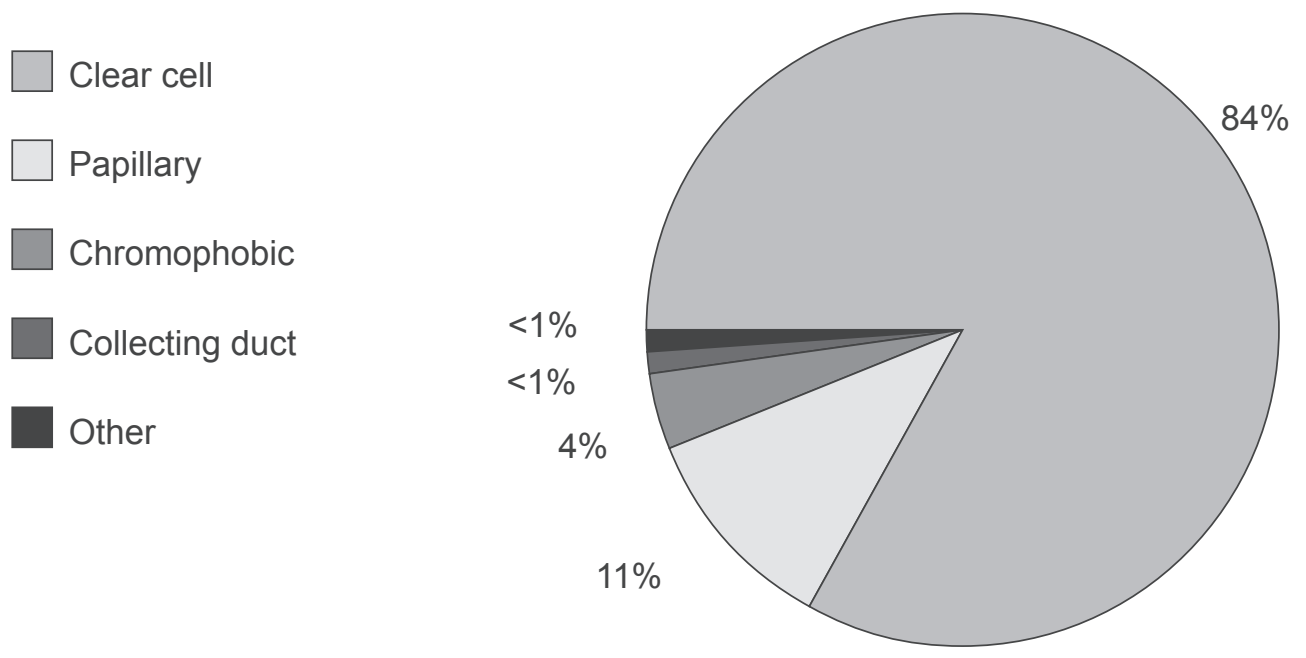

Figure I Clear cell RCC represents the majority of all RCC tumors. Abbreviation: RCC, renal cell carcinoma.

hereditary papillary renal carcinoma (characterized by c-MET proto-oncogene mutations); hereditary leiomyomatosis RCC (characterized by fumarate hydratase gene mutations); Birt-Hogg-Dubé (BHD) syndrome, (characterized by $B H D$ tumor suppressor gene mutation/deletion). Mutations in the $V H L$ gene are also found in sporadic cases of RCC, occurring in $70 \%$ of cases sporadic clear cell RCC (Linehan et al 2003; Vira et al 2007). Considering that clear cell RCC represents $80 \%-85 \%$ of all cases of RCC (Cheville et al 2003), VHL gene mutations are the most frequent genetic aberrations associated with RCC development.

The high degree of vascularization and high expression of VEGF by RCC tumors highlights the fact that RCC is a VEGF-driven disease whose development is directly linked to VEGF overexpression and angiogenesis (Herbst et al 1998; Jacobsen et al 2004; Kaelin 2007). Overexpression of VEGF in RCC results from the excess activity of hypoxiainducible transcription factors such as via hypoxia-inducible factor- $\alpha$ (HIF $\alpha)$ (Kaelin 2005). Under normal oxygen concentrations, the VHL protein controls HIF $\alpha$ expression by targeting it for proteosomal degradation (George and Kaelin 2003; Iliopoulos 2006). However, the formation of VHL protein complexes is suppressed when cells become hypoxic, allowing HIF $\alpha$ expression and the production of pro-angiogenic growth factors such as VEGF. In RCC, mutation or inactivation of the VHL protein is common (Linehan et al 2003; Vira et al 2007) and results in an inability to degrade HIF $\alpha$ in the presence of normal oxygen levels (Kaelin 2007), ultimately leading to increased VEGF expression, angiogenesis and tumor growth. The central role
VEGF plays in the pathophysiology of RCC makes RCC a logical indication for therapy with the direct anti-VEGF inhibitor bevacizumab.

\section{Historical management options in RCC}

Nephrectomy is the primary treatment for patients with RCC presenting with stages I-III disease (tumors limited to the kidney or tumors that extend beyond the kidney, including regional lymph nodes, but without distant metastasis) (Ljungberg et al 2007; National Comprehensive Cancer Network 2008) and can represent a curative option (National Comprehensive Cancer Network 2008). However, approximately $30 \%$ of patients present with advanced and/or metastatic disease (ie, stage IV tumors, which are those that have metastasized or invaded adjacent organs) (Corgna et al 2007) where surgery is usually only palliative. Treatment options for patients who have metastatic disease or are at high risk of recurrence following nephrectomy are limited. RCC is generally refractory to conventional cancer therapies, and as such chemotherapy, radiotherapy and hormone therapy have no established roles in the management of RCC (Corgna et al 2007).

Observations of spontaneous regression of solitary metastases, regression of metastases after nephrectomy, and the presence of lymphocytes infiltrating the primary tumor following IFN and interleukin-2 (IL-2) therapy suggested that these responses may be immune-mediated. IFN and IL-2 are 2 cytokines shown to provide benefit to some patients with RCC (Coppin et al 2005; Yang and Childs 2006). As the only therapeutic options available for patients with advanced and/or metastatic disease, immunotherapy was the standard 
of care for patients with metastatic RCC for approximately 25 years from the 1980s (Porta et al 2007).

Approved in Europe and Japan for metastatic RCC therapy, IFN has immunomodulatory, anti-viral, antiproliferative and anti-angiogenic properties. IL-2 is the only FDA-approved cytokine therapy for metastatic RCC. The overall objective response rate (ORR) with IFN therapy is approximately $15 \%$ (range $0 \%-29 \%$ ) (Fossa 2000) and $17 \%-27 \%$ with high-dose IL-2 therapy (Parton et al 2006; McDermott and Rini 2007). An OS benefit has been demonstrated for IFN (Medical Research Council Renal Cancer Collaborators 1999; Pyrhonen et al 1999). However, the benefit of both of these agents is limited to selected patients, with the Memorial-Sloan Kettering Cancer Center (MSKCC) risk criteria most commonly being used to select patients with good prognosis for therapy (Motzer et al 2002; Motzer et al 2004); a recent trial showed no benefit from IFN therapy in patients with intermediate risk (Negrier et al 2007). Common tolerability issues associated with IFN therapy include fatigue, fever, chills, myalgia and depression (Jonasch and Haluska 2001) and the significant toxicity of high-dose IL-2 regimens limits their use.

\section{How is bevacizumab evolving the way in which we treat patients with metastatic RCC? Phase II trials of bevacizumab in RCC Efficacy}

Two key phase II trials of bevacizumab in RCC have been conducted: AVF0890s (Yang et al 2003) and RACE (Bukowski et al 2007). AVF0890s recruited 116 patients with metastatic RCC who were not optimal candidates for IL-2 therapy or had previously not responded to this therapy. This trial was a randomized, placebo-controlled, double-blind trial of bevacizumab monotherapy. Patients were randomized to 1 of 3 treatment arms: placebo $(n=40)$, bevacizumab $3 \mathrm{mg} / \mathrm{kg}(\mathrm{n}=37)$ or $10 \mathrm{mg} / \mathrm{kg}(\mathrm{n}=39)$. The results from this trial showed that median time to progression (TTP) was significantly longer for the bevacizumab $10 \mathrm{mg} / \mathrm{kg}$ arm than the placebo arm (4.8 vs 2.5 months; hazard ratio $[\mathrm{HR}]=2.55 ; \mathrm{p}<0.001)$ with $10 \%$ of patients achieving a partial response (PR). The median TTP for the bevacizumab $3 \mathrm{mg} / \mathrm{kg}$ arm was not significantly greater than the placebo arm (TTP 3.0 months; HR $=1.26 ; \mathrm{p}=0.053$ ) and no patients achieved a PR. Possibly due to the cross-over of the placebo-treated patients to receive bevacizumab after disease progression, OS was not significantly different between the 3 treatment arms ( $p>0.20$ for all comparisons). The positive results observed with bevacizumab $10 \mathrm{mg} / \mathrm{kg}$ provided the rationale for further studying this dose in RCC.

A substantial number of patients receiving bevacizumab in this trial, while not having sufficient tumor shrinkage to be classified as having a PR or complete response, had mixed tumor responses (Yang 2004). Overall, when patients stopped receiving bevacizumab $10 \mathrm{mg} / \mathrm{kg}$, tumor burden at progression was less than at baseline. The strict criteria for progression (a $25 \%$ increase in the perpendicular diameters of any lesion constituted progression) may have led to premature withdrawal of therapy, even though progression occurred only in a minority of their lesions. These observations highlight the potential benefit of continuing bevacizumab despite limited progression.

The second phase II trial, RACE, evaluated bevacizumab alone or in combination with erlotinib (Tarceva $\left.{ }^{\circledR}\right)$ in patients with metastatic RCC. Erlotinib is a small-molecule tyrosine kinase inhibitor (TKI) of epidermal growth factor receptor (EGFR). EGFR and VEGF are both over expressed in many tumors, and VEGF has been implicated in resistance to EGFR-inhibiting agents (Viloria-Petit et al 2001). RACE was a randomized, double-blind, placebo-controlled trial of 104 patients with metastatic RCC who had received no prior systemic therapy. The treatment arms consisted of bevacizumab $10 \mathrm{mg} / \mathrm{kg}$ every 2 weeks and either erlotinib $150 \mathrm{mg}$ or placebo daily. Treatment continued for a maximum of 24 months or until toxicity or disease progression. A median progression-free survival (PFS) of 8.5 months was achieved with bevacizumab monotherapy; this was not significantly improved by the addition of erlotinib (9.9 months; HR: 0.86; 95\% confidence interval [CI]: 0.50-1.49). The ORR was also similar (13\% with bevacizumab plus placebo vs $14 \%$ with bevacizumab plus erlotinib). One complete response was, however, noted in the bevacizumab plus erlotinib arm. The median survival duration with bevacizumab plus erlotinib was 20 months but median OS with bevacizumab plus placebo had not been reached, possibly due to the greater use of second-line therapies in the bevacizumab only arm compared with the bevacizumab plus erlotinib arm ( $32 \%$ vs $14 \%)$.

\section{Tolerability}

Bevacizumab $10 \mathrm{mg} / \mathrm{kg}$ every 2 weeks as a single agent is generally well tolerated in RCC. In the phase II trials discussed above, bevacizumab therapy was associated with manageable side effects, most commonly hypertension, proteinuria and epistaxis. These events are recognized side effects of bevacizumab based on data from trials in 
various tumor types (Hurwitz et al 2004; Sandler et al 2006; Miller et al 2007). Hypertension was the most common side effect in both studies. In AVF0890s, 36\% of patients ( $20 \%$ grade 3 ) developed hypertension on the bevacizumab $10 \mathrm{mg} / \mathrm{kg}$ arm compared with only $5 \%$ on the placebo arm (no grade 3 events). In the RACE trial, grade 3 hypertension developed in $26 \%$ of patients receiving bevacizumab plus placebo and $31 \%$ bevacizumab plus erlotinib. No grade 4 hypertension was reported. Hypertension was managed with oral antihypertensive medication.

The second most frequent side effect was asymptomatic proteinuria. Grade 3 proteinuria developed in $8 \%$ of patients receiving bevacizumab $10 \mathrm{mg} / \mathrm{kg}$ (AVF0890s trial) and $5.7 \%$ of those receiving bevacizumab plus placebo (RACE trial). In the bevacizumab plus erlotinib arm of the RACE trial, 2 patients developed grade 4 proteinuria. The RACE trial also reported grade $3 / 4$ hemorrhage in $3.8 \%$ of patients in the bevacizumab plus placebo arm and $5.9 \%$ of patients in the bevacizumab plus erlotinib arm. As expected, only patients in the bevacizumab plus erlotinib arm reported grade 3 rash (16\%) and diarrhea (7.8\%).

Long-term treatment with bevacizumab also appears feasible and well tolerated. Four patients from the AVF0890s trial received bevacizumab treatment for 3-5 years, with proteinuria the only significant event attributable to bevacizumab (Yang 2004).

These data indicate that bevacizumab causes side effects typical of inhibition of the VEGF pathway in patients with RCC; similar events are observed at a similar incidence in patients treated with TKIs such as sunitinib, which inhibit VEGF receptors as well as other TKIs, although such agents also cause non-VEGF-specific events such as handfoot syndrome and myelosuppression (Motzer et al 2007). The tolerability of single-agent bevacizumab also created a rationale for trials examining its efficacy and safety in combination with the existing standard of care, which was IFN at the time that phase III trials were initiated.

\section{AVOREN: pivotal phase III trial of bevacizumab, in combination with interferon alpha, in RCC}

Trial design

The efficacy and safety of bevacizumab, in combination with IFN, as first-line therapy was investigated in the ongoing phase III AVOREN (Avastin and Roferon in renal cell carcinoma [BO17705]) trial (Escudier et al 2007b). Bevacizumab was combined with IFN because this was the standard therapy and as earlier clinical trials had suggested that bevacizumab could be combined with other therapies without significantly affecting their tolerability (Hurwitz et al 2004; Sandler et al 2006; Miller et al 2007). In addition, it was believed that using bevacizumab in combination with IFN could maximize patient outcomes because the proposed mechanisms of action of bevacizumab and IFN suggested that these agents may have complementary and synergistic effects (Ferrara et al 2005; Ferrantini et al 2007).

To be considered for inclusion into this trial patients must have had confirmed metastatic RCC of predominantly clear cell histology ( $>50 \%$ clear cell if mixed) and prior nephrectomy for primary RCC. Patients must also have had measurable or non-measurable disease according to RECIST, a Karnofsky performance status (KPS) $\geq 70 \%$ and no proteinuria at baseline $(<0.5 \mathrm{~g}$ of protein in a 24 -hour urine collection). Patients were excluded if they had had prior systemic treatment for metastatic RCC, undergone major surgery within 28 days, had evidence of central nervous system (CNS) metastases or spinal cord compression, uncontrolled hypertension or cardiovascular disease. Patients with evidence of bleeding diathesis or coagulopathy and those receiving full therapeutic doses of oral or parenteral anticoagulants were also ineligible.

Between June 2004 and October 2005, 649 patients with metastatic disease were enrolled. On a 1:1 basis, patients were randomized to receive IFN 9 MIU 3 times a week plus placebo or bevacizumab $10 \mathrm{mg} / \mathrm{kg}$ every 2 weeks plus IFN. IFN therapy was stopped after 52 weeks, but patients could continue to receive single-agent bevacizumab/placebo beyond this time until disease progression. The primary objective of this trial is OS and secondary endpoints included PFS, TTP, and ORR.

\section{Overall efficacy}

An 89\% improvement in median PFS was observed with bevacizumab plus IFN therapy compared with IFN plus placebo (Escudier et al 2007b). The addition of bevacizumab to IFN significantly increased PFS from 5.4 to 10.2 months $(\mathrm{HR}=0.63, \mathrm{p}=0.0001)$. ORR was also improved with bevacizumab plus IFN compared with IFN plus placebo (31\% vs 13\%). The median OS had not been reached in the bevacizumab plus IFN group at the time of reporting. Final OS data are expected at the end of 2008. The median OS in the IFN plus placebo arm is 19.8 months.

\section{Efficacy in patient subgroups}

The addition of bevacizumab to IFN improves PFS in all subgroups analyzed (Bracarda et al 2007a; Melichar et al 2008). 
Patients in both favorable $(\mathrm{n}=180)$ and intermediate $(n=363)$ MSKCC risk groups showed improvements in PFS (median PFS 12.9 vs 7.6 months, HR =0.60; median PFS 10.2 vs 4.5 months, $\mathrm{HR}=0.55$, respectively). Despite patients in the poor MSKCC risk category $(n=54)$ having improved PFS $(\mathrm{HR}=0.81 ; \mathrm{p}=0.457)$, this did not reach statistical significance.

Patients with both clear cell RCC histology ( $\mathrm{n}=564$; median PFS 10.2 vs 5.5 months, HR=0.64; 95\% CI: 0.53-0.77) and mixed RCC histology $(\mathrm{n}=85$; median PFS 5.7 vs 2.9 months, HR $=0.60 ; 95 \%$ CI: $0.33-0.85$ ) had PFS benefit. Significant improvement in PFS was also seen in patients aged $\geq 65$ years $(\mathrm{n}=239 ; \mathrm{HR}=0.77 ; 95 \% \mathrm{CI}$ : $0.58-1.03)$ and $<65$ years $(n=410 ; \mathrm{HR}=0.54 ; 95 \% \mathrm{CI}$ : $0.43-0.68)$. Furthermore, the PFS benefit of bevacizumab plus IFN was observed in patients with reduced kidney function (as assessed by creatinine clearance [CLcr]) (high/normal CLcr: $\mathrm{n}=131$; HR $=0.60$ [95\% CI: 0.46-0.79]; low CLcr: $\mathrm{n}=191 ; \mathrm{HR}=0.65$ [95\% CI: 0.51-0.82]) and VEGF levels below $(\mathrm{HR}=0.44 ; 95 \% \mathrm{CI}$ : 0.32-0.64) and above $(\mathrm{HR}=0.66 ; 95 \% \mathrm{CI}: 0.49-0.93)$ the baseline median. Finally, PFS benefit was observed in patients with either single or multiple metastatic sites treated with bevacizumab to IFN and regardless of whether the sum of baseline tumor measurements was above or below the median. Increased response rates were also observed in all patient subgroups treated with bevacizumab plus IFN.

\section{Overall tolerability}

Both bevacizumab and IFN have predictable and wellestablished tolerability profiles (Jonasch and Haluska 2001; Yang et al 2003; Coppin et al 2005; Bukowski et al 2007) and the tolerability profile for bevacizumab plus IFN in the AVOREN trial is consistent with the side effects previously reported for these agents. Dose intensities (percentage of planned total dose) of bevacizumab/placebo and IFN were similar in the 2 treatment arms $(92 \%$ bevacizumab plus IFN vs $96 \%$ IFN plus placebo for the bevacizumab/placebo arms and $91 \%$ bevacizumab plus IFN vs $96 \%$ IFN plus placebo for the IFN arms). The incidence of grade 3/4 events associated with IFN therapy increased from $15 \%$ to $23 \%$ with the addition of bevacizumab to IFN. However, the duration of IFN therapy was longer in the bevacizumab plus IFN arm compared with the control arm (7.8 vs 4.6 months). The median duration of bevacizumab treatment in the bevacizumab plus IFN arm was 9.7 months in contrast to 5.1 months in the placebo arm. The incidence of bevacizumab-associated grade $3 / 4$ events included hypertension (7\%), proteinuria
$(4 \%)$, bleeding $(3 \%)$, arterial and venous thromboembolic events $(3 \%)$, gastrointestinal perforation $(1 \%)$, and wound healing complications $(<1 \%)$.

\section{Tolerability in patient subgroups}

Analysis of patient subgroups showed that the dose intensity of bevacizumab was similar in subgroups defined by CLcr and by age. The dose intensity of IFN was lower in patients with low CLcr (IFN plus placebo 92\%; bevacizumab plus IFN $78 \%$ ) and $\geq 65$ years (IFN plus placebo $92 \%$; bevacizumab plus IFN 82\%) than in those with normal/high CLcr (IFN plus placebo 99\%; bevacizumab plus IFN 87\%) and $<65$ years (IFN plus placebo $99 \%$; bevacizumab plus IFN 92\%).

In patients in the favorable and intermediate MSKCC risk groups, the incidence of grade $\geq 3$ adverse events (AEs; mean number of AEs per patients) in the bevacizumab plus IFN arm was slightly higher than in the placebo arm: 1.3 and 1.2, respectively, compared with 1.0 and 0.8 in the placebo arm. No difference in the incidence of grade $\geq 3$ AEs was found in patients in the poor MSKCC risk group. A higher incidence of grade $\geq 3$ AEs (bevacizumab 66\%; IFN 48\%) was observed in both treatment arms in patients aged $\geq 65$ years compared with patients $<65$ years (bevacizumab $58 \%$; IFN $45 \%$ ). Patients aged $\geq 65$ years in the bevacizumab arm had a higher incidence of fatigue and asthenia. The overall incidences of grade $\geq 3$ AEs were similar in patients receiving bevacizumab irrespective of kidney performance, with no difference in the incidence of bevacizumab-associated AEs.

Overall, the tolerability of bevacizumab plus IFN was as expected based on data from previous trials of these agents as monotherapy. The tolerability in various subgroups indicates that neither age nor renal function should be used to make decisions on whether to treat patients with bevacizumab plus IFN.

\section{CALGB 90206: a second phase III trial of bevacizumab plus IFN in metastatic RCC}

\section{Trial design}

Cancer and Leukemia Group B (CALGB) trial 90206 is an ongoing phase III trial evaluating the efficacy and safety of adding bevacizumab to IFN in the treatment of metastatic RCC (Rini et al 2008). It is a randomized, open-label study, being conducted in the US by CALGB, a US cooperative group sponsored by the National Cancer Institute. It should be noted that this trial differs from AVOREN in that no placebo was used and the trial is not blinded. 
Eligibility criteria for this study included no prior systemic treatment for RCC, confirmed metastatic RCC with a clear cell component, KPS $\geq 70 \%$, measurable or evaluable disease (by RECIST), adequate end-organ function, no CNS metastases, no uncontrolled hypertension, and no venous thrombosis within 12 months or arterial thrombosis within 6 months. Prior nephrectomy was not a requirement for recruitment to this study.

A total of 732 patients were enrolled on to this trial and randomized to receive bevacizumab $(10 \mathrm{mg} / \mathrm{kg}$ every 2 weeks) plus IFN (9 MIU 3 times a week) or IFN alone. The primary endpoint for this study is OS. Secondary endpoints include PFS, ORR, and safety.

\section{Efficacy and tolerability}

Based on the recommendation of the Data and Safety Monitoring Board (DSMB), a pre-planned interim analysis of the trial results was released. These data confirmed the results of AVOREN, showing that PFS was significantly extended by the addition of bevacizumab to IFN (8.5 months [95\% CI: 7.5-9.7] vs 5.2 months [95\% CI: 3.1-5.6]; $\mathrm{HR}=0.71$ [95\% CI: $0.61-0.83]$ ). The addition of bevacizumab to IFN also improved ORR compared with IFN alone $(25.5 \%$ vs $13.1 \%$ ). The DSMB will continue to monitor OS until the data are mature.

Analyses of patients categorized by their MSKCC risk status showed the addition of bevacizumab to IFN improved PFS in all groups. Significant improvements in PFS were observed in both the favorable $(\mathrm{n}=192)$ and intermediate $(\mathrm{n}=465)$ MSKCC risk groups (median PFS 11.1 vs 5.7 months, $\mathrm{p}=0.0012$; median PFS 8.4 vs 5.3 months, $\mathrm{p}=0.0017$, respectively). Patients in the poor MSKCC risk group ( $\mathrm{n}=75)$ also had improved PFS (3.3 vs 2.6 months), although the difference was not significant $(\mathrm{p}=0.25)$.

CALGB 90206 revealed no new safety signals for the combination of bevacizumab plus IFN regimen (Rini et al 2008). The number of grade $3 / 4$ AEs was higher in the bevacizumab plus IFN arm compared with the IFN arm (79\% vs 61\%), and the most common events were fatigue $(37 \%)$, anorexia $(17 \%)$, proteinuria $(15 \%)$, and hypertension $(10 \%)$.

\section{Comparison with AVOREN}

Both AVOREN and CALGB 90206 show that bevacizumab plus IFN produces significant clinical benefit as first-line therapy for patients with metastatic RCC. Despite differences in median PFS between AVOREN and CALGB 90206, a comparable benefit based on a broad overlap of CIs is observed: CALGB 90206, HR = 0.71 (95\% CI: 0.61-0.83), compared with AVOREN, HR = 0.63 (95\% CI: 0.52-0.75). However, a cross-trial comparison of the absolute results of CALGB 90206 with AVOREN and other trials is inappropriate, as demonstrated by differences in outcomes in the IFN monotherapy control arms in first-line studies (Escudier et al 2007a; Motzer et al 2007). Although AVOREN and CALGB were both phase III trials and used essentially the same treatment regimens, there are a number of potential reasons for the observed differences between the 2 studies.

First, CALGB 90206 was an open-label, cooperative group study whereas AVOREN was a pivotal, double-blind, placebo-controlled, randomized study. The open-label design of CALGB 90206, together with the availability of active second-line therapies (sunitinib and sorafenib) in the US during this trial, may have led to patients discontinuing IFN therapy early, prior to disease progression, and potentially introducing a bias. This is highlighted by the significantly more frequent use of second-line anti-VEGF therapy in both arms of CALGB 90206 compared with AVOREN (bevacizumab plus IFN: $35 \%$ vs $15 \%$; IFN: $48 \%$ vs $20 \%$ ).

A number of differences in patient populations between CALGB 90206 and AVOREN are also evident. First, prior nephrectomy was a requirement for inclusion in the AVOREN trial, but not in CALGB 90206. Patients who have undergone nephrectomy traditionally benefit more from IFN therapy: a meta-analysis by Coppin et al concluded 'nephrectomy followed by interferon-alfa gives the best survival strategy for fully validated therapies' (Coppin et al 2005). Recent data also suggest that patients who have undergone nephrectomy have better outcomes than those who have not when treated with recently approved agents (Szczylik et al 2008). Second, in AVOREN, the proportion of tumor with clear cell histology had to be $>50 \%$; this was not specified in CALGB 90206. As histological subtype is a significant prognostic factor in RCC, this could have impacted on outcomes (Delahunt et al 2007). Third, a greater level of proteinuria was allowed in CALGB 90206 compared with AVOREN (CALGB 90206: <2 g/24 hours; AVOREN: $\leq 0.5 \mathrm{~g} / 24$ hours), potentially allowing the inclusion of patients with a greater degree of renal damage. Taking all of these factors into account, the population in CALGB 90206 may have had a poorer prognosis than that in AVOREN. This is also suggested by the lower than expected efficacy of IFN monotherapy in the favorable MSKCC risk category in CALGB 90206.

Finally, there was a higher incidence of grade 3/4 toxicities in CALGB 90206 compared with AVOREN, with the 
IFN arm having considerably more toxicity in the CALGB 90206 study compared with the IFN plus placebo arm in AVOREN. IFN is not approved by the FDA for the treatment of RCC and is not established as standard of care (IL-2 is the only immunotherapeutic agent approved by the FDA for use in the treatment of patients with RCC). This suggests that limited clinical experience of IFN use in RCC and thus of management of IFN-related toxicities may in part be responsible for the higher incidence of grade 3/4 toxicity with IFN in CALGB 90206. Despite this, no new safety signals were observed in the CALGB 90206 trial.

\section{Summary of clinical experience with bevacizumab in RCC}

Data from phase II and III trials have shown that therapy with bevacizumab is effective and well tolerated in RCC. Recent phase III data from the AVOREN trial have demonstrated that first-line bevacizumab plus IFN produces clinically important and statistically significant improvements in PFS and tumor response compared with IFN alone, irrespective of patient subgroups. Data from CALGB 90206 support the conclusion of the AVOREN trial that bevacizumab plus IFN is an effective first-line combination, producing clinically meaningful benefit to patients with metastatic RCC. Bevacizumab plus IFN is well tolerated and no new toxicities outside of those already known for these 2 therapies have been observed. Bevacizumab and IFN have been used clinically for a number of years and as such their tolerability profiles, as well as side-effect management, are well established.

\section{When selecting first-line therapy, is best PFS the primary goal?}

The approval of a number of new therapies that inhibit angiogenesis has revolutionized the treatment options available for patients with metastatic RCC. During the past few years, 4 new drugs have been approved for use in RCC in the US and/or Europe: bevacizumab (in combination with IFN), sunitinib, sorafenib, and temsirolimus. Because of the rapid pace of change in the treatment environment, a number of questions and challenges remain when selecting the optimal treatment.

When selecting first-line therapy, is best PFS the primary goal? Of the novel agents recently approved for metastatic RCC, the greatest PFS benefits are seen with bevacizumab plus IFN (10.2 months) and sunitinib (11 months). However, a number of patients' needs, individual risks, and diseaserelated factors should be considered when making treatment decisions; for example, patient mobility and profession are important factors in maintaining a patient's quality of life and can influence the choice of primary therapy.

In considering such factors, the specific tolerability of new agents can be used to guide therapy selection to allow treatment to be tailored to the individual circumstances of the patient. Data for bevacizumab in tumors other than RCC indicate that there is a risk of arterial thromboembolic events (ATE) when bevacizumab is used with chemotherapy regimens (Hurwitz et al 2004; Sandler et al 2006; Sugrue et al 2007). Data from phase III trials of bevacizumab in RCC have not revealed an increased risk of ATEs to date (Escudier et al 2007b; Rini et al 2008), but it is appropriate that care should be taken when using bevacizumab in patients with a history of ATEs. Alternatively, sunitinib may be the most suitable therapeutic option for these patients. Sunitinib has been evaluated in patients with brain metastases (Gore et al 2007) and may be more appropriate for this particular patient population as bevacizumab has not been fully investigated in these patients. Similarly, sunitinib's distinct toxicity profile may prevent it being the first choice of therapy in patients with co-morbidities such as nutritional disorders, where the development of diarrhea, nausea and stomatitis/mucositis would be especially undesirable. The risk of immobility and loss of dexterity through the development of hand-foot syndrome with sunitinib therapy may be another factor to consider when deciding how to maintain the patient's daily lifestyle, particularly in relation to the wish to continue to work. Finally, the potentially greater risk of cardiac toxicity in patients treated with sunitinib, which is possibly due to inhibition of multiple factors involved in cardiomyocyte repair and survival in patients with pre-existing cardiac damage (Schmidinger et al 2007), may mean that other options are needed in patients with a history of heart disease. As these particular toxicities are not observed with bevacizumab plus IFN, bevacizumab plus IFN would be the treatment of choice in these patients (Table 1).

The AVOREN trial demonstrated that reducing the dose of IFN can be used to improve further the tolerability of therapy while maintaining efficacy in patients who are unable to tolerate the standard dose of IFN (9 MIU 3 times a week subcutaneously) (Melichar et al 2008). Reducing the dose of IFN significantly reduced the incidence of grade $\geq 3$ AEs (Melichar et al 2008), with a considerable reduction in incidence in the 6-week period after IFN dose reduction compared with the 6 weeks before reduction in patients receiving bevacizumab plus lower-dose IFN (18\% vs 44\%) and lower-dose IFN plus placebo (10\% vs 41\%) (Figure 2). The median PFS in patients receiving bevacizumab plus 
Table I Summary of the adverse events for sunitinib and bevacizumab plus IFN

\begin{tabular}{lll}
\hline All grade (\%) & Sunitinib & Bevacizumab plus IFN \\
\hline Fatigue & 51 & 33 \\
Diarrhea & 53 & 20 \\
Nausea & 44 & $\mathrm{NR}$ \\
Stomatitis & 25 & - \\
Hand-foot syndrome & 20 & - \\
Hypertension & 24 & 26 \\
Bleeding & 12 & 33 \\
Thrombocytopenia & 65 & 6 \\
Anemia & 71 & 10 \\
Proteinuria & - & 18 \\
\hline
\end{tabular}

Abbreviation: IFN, interferon alpha-2a; NR, not reported.

lower-dose IFN was 12.4 months (Figure 3) (Melichar B et al 2007). Response rates were also maintained in the IFN lower-dose population (bevacizumab plus IFN 34\% and IFN plus placebo $17 \%$ vs bevacizumab plus IFN $32 \%$ and IFN plus placebo $13 \%$ in the total population) (Melichar et al 2008). This indicates that IFN dose reduction allows tolerability to be managed while the efficacy of the bevacizumab plus
IFN regimen is maintained. Improvements in tolerability by reducing the dose of IFN are also observed with sorafenib (Bracarda et al 2007b; Gollob et al 2007; Ryan et al 2007).

In summary, a number of parameters, such as patients' needs, individual risks and disease-related factors, as well as efficacy should all be considered when making treatment decisions. The tolerability profiles of novel therapies can help guide first-line therapy to offer bespoke treatment options to suit the needs of the individual patient. The combination of bevacizumab plus IFN provides physicians with a flexible treatment regimen for metastatic RCC and allows the reduction of IFN dose to improve tolerance and manage toxicity without compromising efficacy.

\section{Future strategies for providing additional benefit to patients}

Can combination therapy offer greater benefit to patients than one agent alone? With the approval of a number of novel therapies for treatment of RCC, the next logical step is to combine them and assess their interaction. Combining direct anti-VEGF therapy such as bevacizumab with multi-targeted TKIs may increase anti-tumor efficacy by maximizing inhibition of the VEGF pathway (Sosman et al 2007).

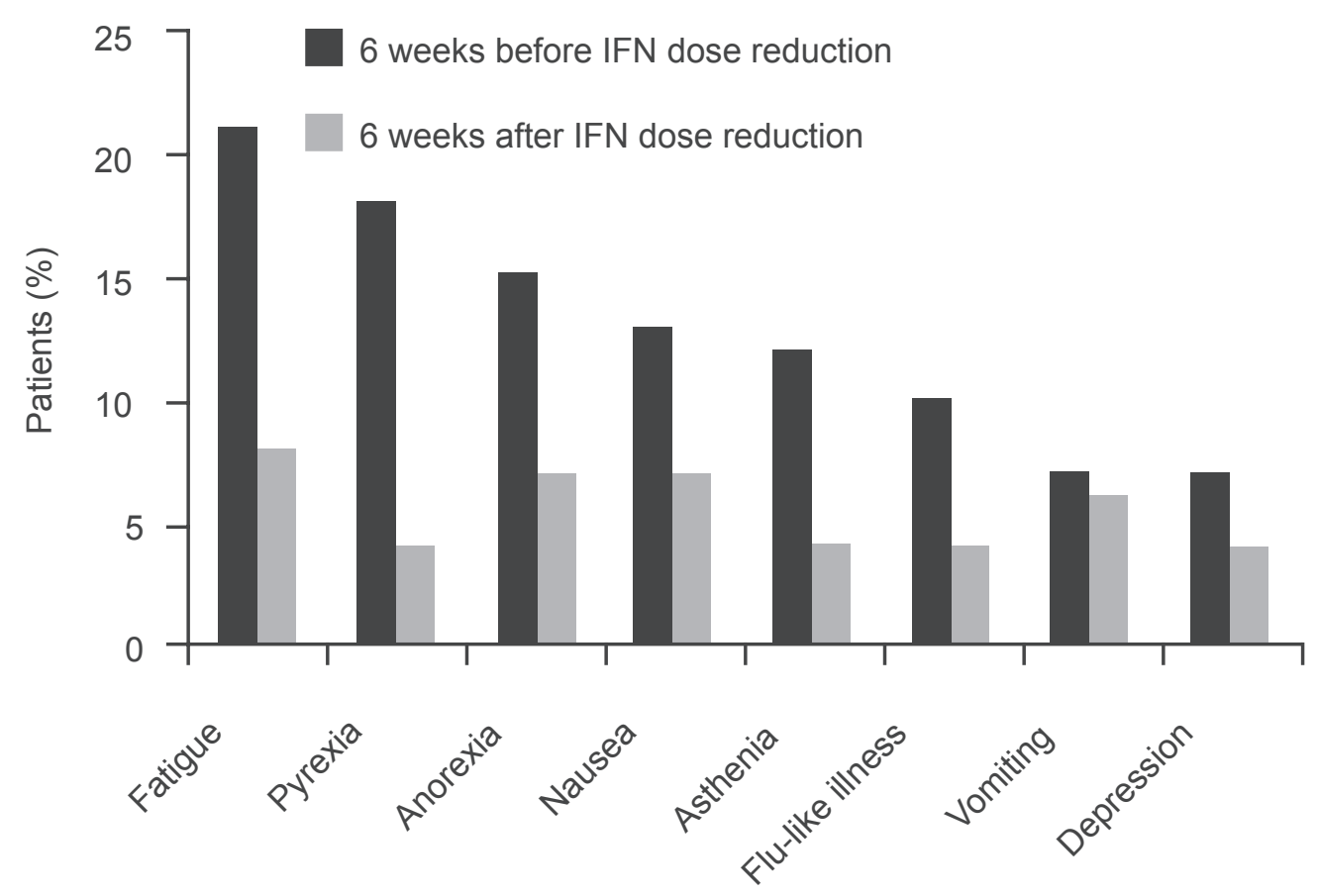

Figure $\mathbf{2}$ Lowering the dose of IFN improves tolerability. Abbreviation: IFN, interferon alpha-2a. 


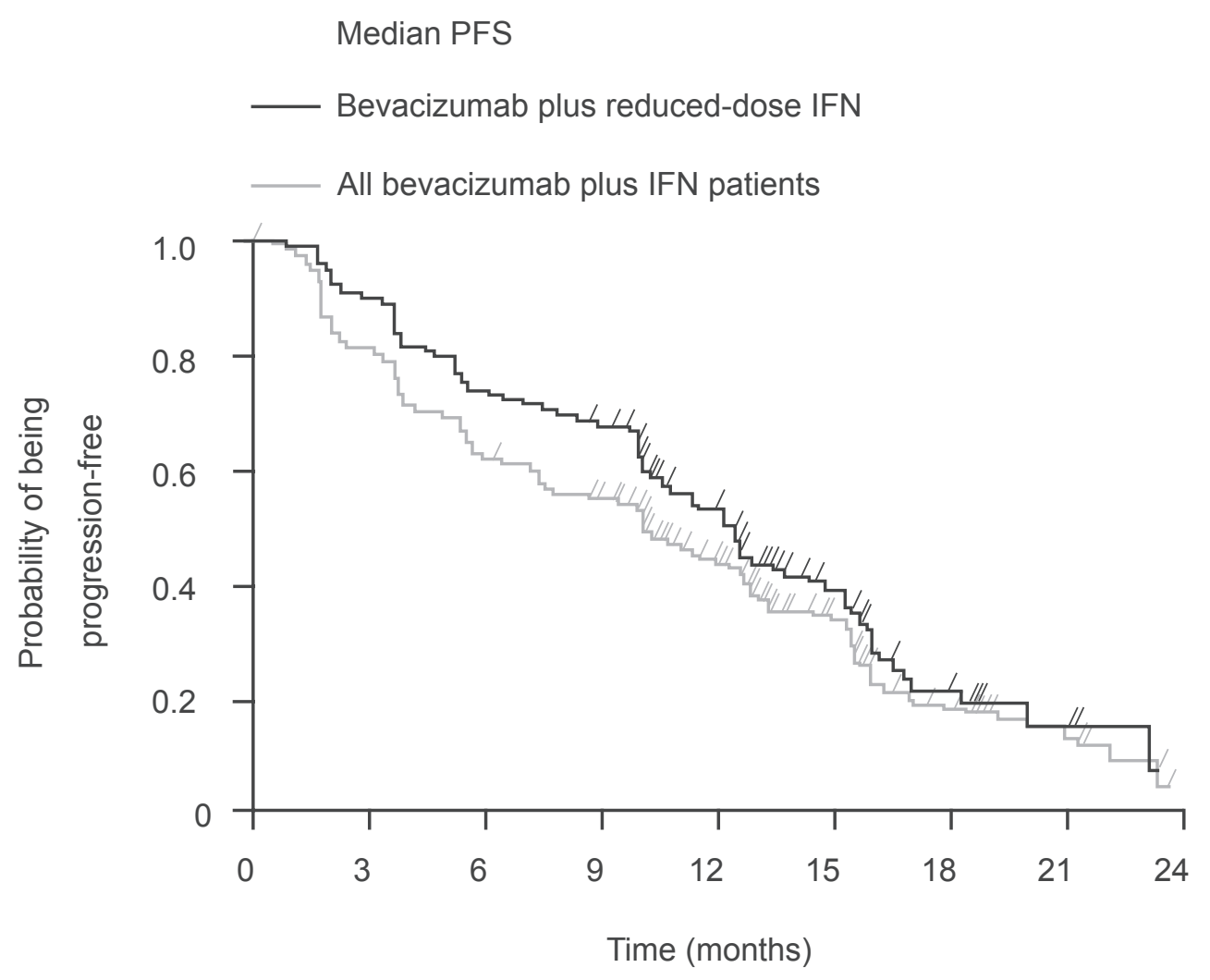

Figure 3 Bevacizumab plus lower-dose IFN has a similar PFS to the total study population receiving bevacizumab plus IFN Abbreviations: PFS, progression-free survival; IFN, interferon alpha-2a.

However, tolerability has to be considered when designing combinations of different therapies.

Although data to date show that objective responses can be achieved by combining bevacizumab with TKIs, an increase in the incidence and severity of TKI associated toxicity is also observed. A phase I trial assessing the safety and maximum tolerated dose of bevacizumab plus sunitinib revealed an ORR of $52 \%(\mathrm{n}=25)$ (Feldman et al 2008), higher than that observed with either bevacizumab plus IFN or sunitinib (Escudier et al 2007b; Motzer et al 2007). The maximum tolerated dose of bevacizumab and sunitinib determined by this trial was $10 \mathrm{mg} / \mathrm{kg}$ intravenously every 2 weeks and sunitinib $50 \mathrm{mg} /$ day for 4 of every 6 weeks. Hypertension was the most frequent grade $3 / 4$ event and 2 dose-limiting toxicities (DLTs) occurred (grade 4 hemorrhage). To manage toxicity, sunitinib dose reduction was required in $40 \%$ of patients. This study concluded that while bevacizumab plus sunitinib was active, this combination was poorly tolerated at full doses as a high proportion of patients experienced toxicity requiring sunitinib dose reductions and/or study discontinuation. This conclusion was echoed in the phase II SABRE-R trial of bevacizumab plus sunitinib where the same regimen was studied. This trial was stopped due to the level of toxicity that was observed with long-term therapy, suggesting that this combination cannot be recommended for further trial. In contrast to these 2 studies, Cooney et al report bevacizumab plus sunitinib is tolerable at full doses without unexpected toxicities (Cooney et al 2008). This phase I trial investigates various dose combinations of bevacizumab and sunitinib in multiple solid tumors. A PR of $30 \%$ is reported in the total study population evaluable for response $(n=23)$. One patient experienced a DLT (grade 4 hypertension) with $37.5 \mathrm{mg}$ sunitinib and $5 \mathrm{mg} / \mathrm{kg}$ bevacizumab, with grade 3 hypertension occurring in 13 other patients.

Combining bevacizumab plus sorafenib showed a PR rate of $46 \%(n=46)$, with a TTP of 11.2 months (Sosman et al 2008). However, sorafenib-associated DLTs (hand-foot syndrome and anorexia) prevented full-dose combination therapy. As combination of lower doses of these agents appears both feasible and to have high activity, further studies are being performed (see below).

Temsirolimus inhibits mammalian target of rapamycin (mTOR) signaling as opposed to bevacizumab, sunitinib and sorafenib which inhibit the VEGF pathway. This different mechanism of action suggests that it should be combinable with bevacizumab and other agents. However, a phase I trial 
indicated that sunitinib plus temsirolimus has unacceptable toxicity (Fischer et al 2008). In contrast, a dose-escalation trial of bevacizumab $(5-10 \mathrm{mg} / \mathrm{kg}$ every 2 weeks) plus temsirolimus (10-25 mg weekly) showed that 8 of 12 patients had PRs, for an ORR of $67 \%$ (Merchan et al 2007). A further 3 patients had stable disease as their best response, for a clinical benefit rate of $92 \%$. Two DLTs occurred (grade 3 hypertriglyceridemia, grade 3 stomatitis), but no grade 4 toxicities related to therapy occurred. The combinability of bevacizumab with mTOR inhibitors was further demonstrated by the phase II trial of bevacizumab in combination with everolimus (RAD001) (Whorf et al 2008). Patients received bevacizumab $10 \mathrm{mg} / \mathrm{kg}$ every 2 weeks and everolimus $10 \mathrm{mg}$ daily. An objective response of $21 \%$ and a minor response/stable disease of $69 \%$ was observed in the $90 \%$ of patients who completed 8 weeks of treatment. Grade 3/4 proteinuria occurred in 10 patients $(19 \%)$ but other grade $3 / 4$ toxicity was uncommon (fatigue $9 \%$, stomatitis $8 \%$ ). These data suggest that the combination of bevacizumab with everolimus is active and tolerable treatment for metastatic clear cell RCC.

The data described above suggest bevacizumab can be combined with a range of novel agents (Figure 4). However, trials in which sunitinib or sorafenib have been combined with other agents show that combinations including these agents are poorly tolerated or that significant dose reduction is required to ensure tolerability, with considerable loss of efficacy (Sosman et al 2006; Jonasch et al 2007; Kondagunta et al 2007; Ryan et al 2007).

Larger, more robust studies are required to investigate whether combinations of novel agents will offer true benefit to patients with metastatic RCC. Bevacizumab in combination with sorafenib or temsirolimus is currently being studied in 3 trials of combination therapy in RCC. The BeST trial is a 4-arm, randomized phase II trial that will compare the efficacy and safety of bevacizumab, sorafenib and temsirolimus combinations to those of bevacizumab alone. A second trial, trial 3311, is a 2-arm phase III trial comparing bevacizumab plus IFN with bevacizumab plus temsirolimus in 822 patients. TORAVA, a French phase II trial, is ongoing and will compare sunitinib with bevacizumab plus IFN and bevacizumab plus temsirolimus.

Because novel therapies target different pathways, it is possible that sequential use of agents may have further efficacy following disease progression. This strategy is being investigated by several phase I/II trials to identify the optimal sequence of therapy that can improve patient outcomes.

\begin{tabular}{|c|c|c|c|c|c|}
\hline \multirow[b]{2}{*}{ Agent } & \multicolumn{5}{|c|}{ Combination partner } \\
\hline & Bevacizumab & Sunitinib & Sorafenib & Temsirolimus & Cytokines \\
\hline Bevacizumab & & $x$ & $\begin{array}{c}S / ? \\
\text { (dose } \\
\text { reduction } \\
\text { required) }\end{array}$ & $\checkmark$ & $\checkmark$ \\
\hline Sunitinib & $x$ & & NK & $x$ & $\begin{array}{c}\delta / ? \\
\text { (dose } \\
\text { reduction } \\
\text { required } \\
\text { for both) }\end{array}$ \\
\hline Sorafenib & $\begin{array}{l}\mathcal{J} / ? \\
\text { (dose } \\
\text { reduction } \\
\text { required) }\end{array}$ & NK & & NK & $\begin{array}{l}\quad \mathcal{J} / ? \\
\text { (IFN dose } \\
\text { reduction } \\
\text { required) }\end{array}$ \\
\hline Temsirolimus & $\checkmark$ & $X$ & NK & & $\begin{array}{c}\checkmark \\
\text { (no } \\
\text { additional } \\
\text { benefit) }\end{array}$ \\
\hline
\end{tabular}

Figure 4 Bevacizumab is a good partner for combination therapy. Abbreviations: NK, not known; IFN, interferon alpha-2a. 
In patients who become refractory to bevacizumab therapy, sunitinib and sorafenib appear to be effective as second-line agents (Drabkin et al 2007; Hutson et al 2007). Sunitinib following first-line bevacizumab achieved an ORR in 61 patients of 23\% (14 PRs, 36 stable diseases), with a PFS of 30 weeks (Hutson et al 2007). The most common treatment-related AEs were fatigue, diarrhea, and nausea. A subgroup analysis of patients enrolled on the sorafenib expanded access trial who had received prior bevacizumab therapy showed that stable disease was achieved in $77 \%$ of patients $(n=197)$ treated with sorafenib (Drabkin et al 2007). The most frequent grade $3 / 4$ toxicities were hand-foot syndrome, fatigue and hypertension. The incidence of AEs leading to dose interruption, reduction, or discontinuation of sorafenib was $39 \%, 20 \%$, and $26 \%$ respectively.

\section{Summary of bevacizumab in RCC}

Data from 2 large phase III trials show that bevacizumab is an effective first-line therapy for the treatment of metastatic RCC, offering significant extension in PFS and improved response rate when used in combination with IFN. Bevacizumab plus IFN is well tolerated, with dose reduction of IFN providing the flexibility to improve tolerability further. The overall tolerability profile of bevacizumab plus IFN in comparison with other agents available for first-line treatment of RCC is important when tailoring therapy for individual patients.

With a number of novel therapies approved for the treatment of RCC, it will be vital to optimize combination and/or sequencing strategies to ensure that the use of all available therapies is optimized to maximize benefit for patients with metastatic RCC. Data to date and tolerability profiles favor bevacizumab as a combination partner and, consequently, randomized trials have been initiated to address bevacizumab's potential. Before starting first-line therapy, the complete sequence of treatment should also be considered. Current data suggest that bevacizumab has maximal benefit when used first line (Bukowski et al 2007; Escudier et al 2007b) and, therefore, approaches based on this observation, with bevacizumab administered on diagnosis of metastatic RCC and other agents administered on progression, may be reasonable. Bevacizumab has a central role to play in future advances in RCC therapy.

\section{Acknowledgments}

The authors acknowledge the medical writing assistance of Stuart Langley (Gardiner-Caldwell Communications, Macclesfield, UK).

\section{Disclosures}

JC and SJ are employees of F. Hoffmann-La Roche Ltd. BE has no conflicts of interest to disclose.

\section{References}

Bloch W, Huggel K, Sasaki T, et al. 2000. The angiogenesis inhibitor endostatin impairs blood vessel maturation during wound healing. FASEB J, 14:2373-6.

Bosetti C, Bertuccio P, Levi F, et al. 2008. Cancer mortality in the European Union, 1970-2003, with a joinpoint analysis. Ann Oncol, 19:631-40.

Bracarda S, Koralewski R, Pluzanska A, et al. 2007a. Bevacizumab/ interferon-alpha2a provides a progression-free survival benefit in all prespecified patient subgroups as first-line treatment of metastatic renal cell carcinoma (AVOREN) [abstract]. Eur J Cancer Suppl, 5:281-2.

Bracarda S, Porta C, Boni C, et al. 2007b. Randomized prospective phase II trial of two schedules of sorafenib daily and interferon-a2a (IFN) in metastatic renal cell carcinoma (RAPSODY): GOIRC Study 0681 [abstract]. J Clin Oncol, 25 Suppl:259s.

Bukowski RM, Kabbinavar FF, Figlin RA, et al. 2007. Randomized phase II study of erlotinib combined with bevacizumab compared with bevacizumab alone in metastatic renal cell cancer. J Clin Oncol, 25:4536-41.

Carmeliet P. 2005. VEGF as a key mediator of angiogenesis in cancer. Oncology, 69 (Suppl 3):4-10.

Carmeliet P, Ferreira V, Breier G, et al. 1996. Abnormal blood vessel development and lethality in embryos lacking a single VEGF allele. Nature, 380:435-9.

Cheville JC, Lohse CM, Zincke H, et al. 2003. Comparisons of outcome and prognostic features among histologic subtypes of renal cell carcinoma. Am J Surg Pathol, 27:612-24.

Cooney MM, Garcia J, Elson P, et al. 2008. Sunitinib and bevacizumab in advanced solid tumors: A phase I trial [abstract]. J Clin Oncol, 26(Suppl):160s.

Coppin C, Porzsolt F, Awa A, et al. 2005. Immunotherapy for advanced renal cell cancer. Cochrane Database Syst Rev, CD001425.

Corgna E, Betti M, Gatta G, et al. 2007. Renal cancer. Crit Rev Oncol Hematol, 64:247-62.

Delahunt B, Bethwaite PB, Nacey JN. 2007. Outcome prediction for renal cell carcinoma: evaluation of prognostic factors for tumours divided according to histological subtype. Pathology, 39:459-65.

Drabkin HA, Figlin R, Stadler WM, et al. 2007. The advanced renal cell carcinoma sorafenib (ARCCS) expanded access trial: safety and efficacy in patients (pts) with prior bevacizumab (BEV) treatment [abstract]. $J$ Clin Oncol, 25(Suppl):245s.

Escudier B, Eisen T, Stadler WM, et al. 2007a. Sorafenib in advanced clear-cell renal-cell carcinoma. $N$ Engl J Med, 356:125-34.

Escudier B, Pluzanska A, Koralewski P, et al. 2007b. Bevacizumab plus interferon alfa-2a for treatment of metastatic renal cell carcinoma: a randomised, double-blind phase III trial. Lancet, 370:2103-11.

Feldman DR, Ginsberg M, Baum CM, et al. 2008. Phase I trial of bevacizumab plus sunitinib in patients with metastatic renal cell carcinoma [abstract]. J Clin Oncol, 26(Suppl):274s.

Ferlay J, Autier P, Boniol M, et al. 2007. Estimates of the cancer incidence and mortality in Europe in 2006. Ann Oncol, 18:581-92.

Ferrantini M, Capone I, Belardelli F. 2007. Interferon-alpha and cancer: mechanisms of action and new perspectives of clinical use. Biochimie, 89:884-93.

Ferrara N. 2004. Vascular endothelial growth factor: basic science and clinical progress. Endocr Rev, 25:581-611.

Ferrara N, Chen H, vis-Smyth T, et al. 1998. Vascular endothelial growth factor is essential for corpus luteum angiogenesis. Nat Med, 4:336-40.

Ferrara N, Gerber HP, LeCouter J. 2003. The biology of VEGF and its receptors. Nat Med, 9:669-76. 
Ferrara N, Henzel WJ. 1989. Pituitary follicular cells secrete a novel heparin-binding growth factor specific for vascular endothelial cells. Biochem Biophys Res Commun, 161:851-8.

Ferrara N, Hillan KJ, Novotny W. 2005. Bevacizumab (Avastin), a humanized anti-VEGF monoclonal antibody for cancer therapy. Biochem Biophys Res Commun, 333:328-35.

Fischer P, Patel PH, Carducci M, et al. 2008. Phase I study combining treatment with temsirolimus and sunitinib malate in patients with advanced renal cell carcinoma [abstract]. J Clin Oncol, 26(Suppl):672s.

Folkman J. 1971. Tumor angiogenesis: therapeutic implications. $N$ Engl $J$ Med, 285:1182-6.

Folkman J. 2005. Antiangiogenesis agents. In: DeVita VT, Helmann S, Rosenberg SA (eds) Cancer Principles and Practice of Oncology. 7th ed. Philadelphia: Lippincott Williams and Wilkins. p 2865-82.

Folkman J, Merler E, Abernathy C, et al. 1971. Isolation of a tumor factor responsible for angiogenesis. J Exp Med, 133:275-88.

Fossa SD. 2000. Interferon in metastatic renal cell carcinoma. Semin Oncol, 27:187-93

George DJ, Kaelin WG. 2003. The von Hippel-Lindau protein, vascular endothelial growth factor, and kidney cancer. $N$ Engl $J$ Med, 349:419-21.

Gerber HP, Vu TH, Ryan AM, et al. 1999. VEGF couples hypertrophic cartilage remodeling, ossification and angiogenesis during endochondral bone formation. Nat Med, 5:623-8.

Gollob JA, Rathmell WK, Richmond TM, et al. 2007. Phase II trial of sorafenib plus interferon alfa- $2 \mathrm{~b}$ as first- or second-line therapy in patients with metastatic renal cell cancer. J Clin Oncol, 25:3288-95.

Gordon MS, Margolin K, Talpaz M, et al. 2001. Phase I safety and pharmacokinetic study of recombinant human anti-vascular endothelial growth factor in patients with advanced cancer. J Clin Oncol, 19:843-50.

Gore M, Szczylik C, Porta C, et al. 2007. Sunitinib in metastatic renal cell carcinoma (mRCC): preliminary assessment of safety and efficacy in an expanded access trial with subpopulation analysis [abstract] Eur J Cancer Suppl, 5:299.

Herbst C, Kosmehl H, Stiller KJ, et al. 1998. Evaluation of microvessel density by computerised image analysis in human renal cell carcinoma. Correlation to $\mathrm{pT}$ category, nuclear grade, proliferative activity and occurrence of metastasis. J Cancer Res Clin Oncol, 124:141-7.

Hurwitz H, Fehrenbacher L, Novotny W, et al. 2004. Bevacizumab plus irinotecan, fluorouracil, and leucovorin for metastatic colorectal cancer. N Engl J Med, 350:2335-42.

Hutson TE, George DJ, Michaelson MD, et al. 2007. A phase 2 trial of sunitinib in bevacizumab-refractory metastatic renal cell carcinoma (mRCC): updated results and analysis of circulating biomarkers [abstract]. Eur J Cancer Suppl, 5:301.

Iliopoulos O. 2006. Molecular biology of renal cell cancer and the identification of therapeutic targets. J Clin Oncol, 24:5593-600.

Jacobsen J, Grankvist K, Rasmuson T, et al. 2004. Expression of vascular endothelial growth factor protein in human renal cell carcinoma BJU Int, 93:297-302.

Jain RK. 2002. Tumor angiogenesis and accessibility: role of vascular endothelial growth factor. Semin Oncol, 29:3-9.

Jemal A, Siegel R, Ward E, et al. 2008. Cancer statistics, 2008. CA Cancer J Clin, 58:71-96.

Johnson DH, Fehrenbacher L, Novotny WF, et al. 2004. Randomized phase II trial comparing bevacizumab plus carboplatin and paclitaxel with carboplatin and paclitaxel alone in previously untreated locally advanced or metastatic non-small-cell lung cancer. J Clin Oncol, 22:2184-91.

Jonasch E, Corn P, Ashe RG, et al. 2007. Randomized phase II study of sorafenib with or without low-dose IFN in patients with metastatic renal cell carcinoma [abstract]. J Clin Oncol, 25 Suppl:260s.

Jonasch E, Haluska FG. 2001. Interferon in oncological practice: review of interferon biology, clinical applications, and toxicities. Oncologist, $6: 34-55$.
Kabbinavar F, Hurwitz HI, Fehrenbacher L, et al. 2003. Phase II, randomized trial comparing bevacizumab plus fluorouracil (FU)/leucovorin (LV) with $\mathrm{FU} / \mathrm{LV}$ alone in patients with metastatic colorectal cancer. $J$ Clin Oncol, 21:60-5.

Kaelin WG. 2005. The von Hippel-Lindau protein, HIF hydroxylation, and oxygen sensing. Biochem Biophys Res Commun, 338:627-38.

Kaelin WG. 2007. The von Hippel-Lindau tumor suppressor protein and clear cell renal carcinoma. Clin Cancer Res, 13:680s-4s.

Karim-Kos HE, de VE, Soerjomataram I, et al. 2008. Recent trends of cancer in Europe: A combined approach of incidence, survival and mortality for 17 cancer sites since the 1990s. Eur J Cancer, 44:1345-89.

Kim KJ, Li B, Houck K, et al. 1992. The vascular endothelial growth factor proteins: identification of biologically relevant regions by neutralizing monoclonal antibodies. Growth Factors, 7:53-64.

Kim KJ, Li B, Winer J, et al. 1993. Inhibition of vascular endothelial growth factor-induced angiogenesis suppresses tumour growth in vivo. Nature, 362:841-4.

Kondagunta GV, Hudes G, Figlin R, et al. 2007. Sunitinib malate (SU) plus interferon (IFN) in first line metastatic renal cell cancer (mRCC): Results of a dose-finding study [abstract]. J Clin Oncol, 25(Suppl):260s.

Leung DW, Cachianes G, Kuang WJ, et al. 1989. Vascular endothelial growth factor is a secreted angiogenic mitogen. Science, 246:1306-9.

Levi F, Ferlay J, Galeone C, et al. 2008. The changing pattern of kidney cancer incidence and mortality in Europe. BJU Int, 101:949-58.

Linehan WM, Walther MM, Zbar B. 2003. The genetic basis of cancer of the kidney. $J$ Urol, 170:2163-72.

Lipworth L, Tarone RE, McLaughlin JK. 2006. The epidemiology of renal cell carcinoma. J Urol, 176:2353-8.

Ljungberg B, Hanbury DC, Kuczyk MA, et al. 2007. Renal cell carcinoma guideline. Eur Urol, 51:1502-10.

Mancuso MR, Davis R, Norberg SM, et al. 2006. Rapid vascular regrowth in tumors after reversal of VEGF inhibition. J Clin Invest, 116:2610-21.

Margolin K, Gordon MS, Holmgren E, et al. 2001. Phase Ib trial of intravenous recombinant humanized monoclonal antibody to vascular endothelial growth factor in combination with chemotherapy in patients with advanced cancer: pharmacologic and long-term safety data. J Clin Oncol, 19:851-6.

McDermott DF, Rini BI. 2007. Immunotherapy for metastatic renal cell carcinoma. BJU Int, 99:1282-8.

Medical Research Council Renal Cancer Collaborators. 1999. Interferonalpha and survival in metastatic renal carcinoma: early results of a randomised controlled trial. Lancet, 353:14-7.

Melichar B, Kowalski J, Pluzanska A, et al. 2007. First-line bevacizumab improves progression-free survival with reduced doses of interferona2a in the treatment of patients with metastatic renal cell carcinoma (AVOREN) [abstract]. Eur J Cancer Suppl, 5:304.

Melichar B, Koralewski P, Ravaud A, et al. 2008. First-line bevacizumab combined with reduced dose interferon-\{alpha $2 \mathrm{a}$ is active in patients with metastatic renal cell carcinoma. Ann Oncol, 19:1470-6.

Merchan JR, Liu G, Fitch T, et al. 2007. Phase I/II trial of CCI-779 and bevacizumab in stage IV renal cell carcinoma: phase I safety and activity results [abstract]. J Clin Oncol, 25 Suppl:243s.

Miller K, Wang M, Gralow J, et al. 2007. Paclitaxel plus bevacizumab versus paclitaxel alone for metastatic breast cancer. $N \mathrm{Engl} \mathrm{J} \mathrm{Med,}$ 357:2666-76.

Motzer RJ, Bacik J, Murphy BA, et al. 2002. Interferon-alfa as a comparative treatment for clinical trials of new therapies against advanced renal cell carcinoma. J Clin Oncol, 20:289-96.

Motzer RJ, Bacik J, Schwartz LH, et al. 2004. Prognostic factors for survival in previously treated patients with metastatic renal cell carcinoma. $J$ Clin Oncol, 22:454-63.

Motzer RJ, Bander NH, Nanus DM. 1996. Renal-cell carcinoma. N Engl $J$ Med, 335:865-75. 
Motzer RJ, Hutson TE, Tomczak P, et al. 2007. Sunitinib versus interferon alfa in metastatic renal-cell carcinoma. $N$ Engl J Med, 356:115-24.

National Comprehensive Cancer Network. 2008. Clinical practice guidelines in oncology. Version 1. Accessed August 2008. URL: http://www.nccn. org/professionals/physician_gls/PDF/kidney pdf.

Negrier S, Perol D, Ravaud A, et al. 2007. Medroxyprogesterone, interferon alfa-2a, interleukin 2, or combination of both cytokines in patients with metastatic renal carcinoma of intermediate prognosis: results of a randomized controlled trial. Cancer, 110:2468-77.

Parton M, Gore M, Eisen T. 2006. Role of cytokine therapy in 2006 and beyond for metastatic renal cell cancer. J Clin Oncol, 24:5584-92.

Porta C, Paglino C, Imarisio I, et al. 2007. Cytokine-based immunotherapy for advanced kidney cancer: past results and future perspectives in the era of molecularly targeted agents. Scientific World Journal, 7:837-49.

Presta LG, Chen H, O'Connor SJ, et al. 1997. Humanization of an antivascular endothelial growth factor monoclonal antibody for the therapy of solid tumors and other disorders. Cancer Res, 57:4593-9.

Pyrhonen S, Salminen E, Ruutu M, et al. 1999. Prospective randomized trial of interferon alfa-2a plus vinblastine versus vinblastine alone in patients with advanced renal cell cancer. J Clin Oncol, 17:2859-67.

Rini B, Halabi S, Rosenberg J, et al. 2008. CALGB 90206: a phase III trial of bevacizumab plus interferon-alpha monotherapy in metastatic renal cell carcinoma [online] [abstract]. Presented at: Genitourinary Cancers Symposium 14-16 February. Accessed August 2008. URL: http://www.asco.org/ASCO/Abstracts+\%26+Virtual+Meeting/ Abstracts? \&vmview=abst_detail_view\&confID=54\&abstractID $=2$ 0239.

Roche Media News. 2007. Avastin approved in Europe for first-line treatment of patients with advanced kidney cancer [online]. Accessed August 2008. URL: http://www.roche.com/med-cor-2007-12-19.

Ryan CW, Goldman BH, Lara PN, et al. 2007. Sorafenib with interferon alfa- $2 \mathrm{~b}$ as first-line treatment of advanced renal carcinoma: a phase II study of the Southwest Oncology Group. J Clin Oncol, 25:3296-301.

Sandler A, Gray R, Perry MC, et al. 2006. Paclitaxel-carboplatin alone or with bevacizumab for non-small-cell lung cancer. $N$ Engl $J$ Med, 355:2542-50.

Schmidinger M, Vogl UM, Schukro C, et al. 2007. Cardiac involvement in patients with sorafenib or sunitinib treatment for metastatic renal cell carcinoma [abstract]. J Clin Oncol, 25(Suppl):262s.

Senger DR, Galli SJ, Dvorak AM, et al. 1983. Tumor cells secrete a vascular permeability factor that promotes accumulation of ascites fluid. Science, 219:983-5.

Senger DR, Perruzzi CA, Feder J, et al. 1986. A highly conserved vascular permeability factor secreted by a variety of human and rodent tumor cell lines. Cancer Res, 46:5629-32.
Sosman J, Flaherty K, Atkins M, et al. 2008. Updated results of phase I trial of sorafenib (S) and bevacizumab (B) in patients with metastatic renal cell cancer (mRCC) [abstract]. J Clin Oncol, 26:252s.

Sosman JA, Flaherty K, Atkins MB, et al. 2006. A phase I/II trial of sorafenib (S) with bevacizumab (B) in metastatic renal cell cancer (mRCC) patients (pts) [abstract]. J Clin Oncol, 24:128s.

Sosman JA, Puzanov I, Atkins MB. 2007. Opportunities and obstacles to combination targeted therapy in renal cell cancer. Clin Cancer Res, 13:764S-9S.

Sugrue MM, Yi D, Purdie D, et al. 2007. Serious arterial thromboembolic events (sATE) in patients (pts) with metastatic colorectal cancer (mCRC) treated with bevacizumab (BV): Results from the BRiTE registry [abstract]. J Clin Oncol, 25:197s.

Szczylik C, Porta C, Bracarda S, et al. 2008. Sunitinib in patients with or without prior nephrectomy (Nx) in an expanded access trial of metastatic renal cell carcinoma (mRCC) [abstract]. J Clin Oncol, 26:280s.

Tong RT, Boucher Y, Kozin SV, et al. 2004. Vascular normalization by vascular endothelial growth factor receptor 2 blockade induces a pressure gradient across the vasculature and improves drug penetration in tumors. Cancer Res, 64:3731-6.

Viloria-Petit A, Crombet T, Jothy S, et al. 2001. Acquired resistance to the antitumor effect of epidermal growth factor receptor-blocking antibodies in vivo: a role for altered tumor angiogenesis. Cancer Res, 61:5090-101.

Vira MA, Novakovic KR, Pinto PA, et al. 2007. Genetic basis of kidney cancer: a model for developing molecular-targeted therapies. BJU Int, 99:1223-9.

Warren RS, Yuan H, Matli MR, et al. 1995. Regulation by vascular endothelial growth factor of human colon cancer tumorigenesis in a mouse model of experimental liver metastasis. J Clin Invest, 95:1789-97.

Whorf RC, Hainsworth J, Spigel DR, et al. 2008. Phase II study of bevacizumab and everolimus (RAD001) in the treatment of advanced renal cell carcinoma (RCC) [abstract]. J Clin Oncol, 26:252s.

Wildiers H, Guetens G, De BG, et al. 2003. Effect of antivascular endothelial growth factor treatment on the intratumoral uptake of CPT-11. Br J Cancer, 88:1979-86.

Willett CG, Boucher Y, di Tomaso E, et al. 2004. Direct evidence that the VEGF-specific antibody bevacizumab has antivascular effects in human rectal cancer. Nat Med, 10:145-7.

Yang JC. 2004. Bevacizumab for patients with metastatic renal cancer: an update. Clin Cancer Res, 10:6367S-70S.

Yang JC, Childs R. 2006. Immunotherapy for renal cell cancer. J Clin Oncol, 24:5576-83.

Yang JC, Haworth L, Sherry RM, et al. 2003. A randomized trial of bevacizumab, an anti-vascular endothelial growth factor antibody, for metastatic renal cancer. $N$ Engl J Med, 349:427-34. 